\title{
Control of turbulence in oscillatory reaction-diffusion systems through a combination of global and local feedback
}

\author{
Michael Stich* \\ Centro de Astrobiología (CSIC-INTA), Instituto Nacional de Técnica Aeroespacial, Ctra de Ajalvir km 4, \\ 28850 Torrejón de Ardoz, Madrid, Spain \\ Alfonso C. Casal \\ Department of Applied Mathematics, E.T.S. Arquitectura, Universidad Politécnica de Madrid, Avda. Juan de Herrera, \\ 28040 Madrid, Spain \\ Jesús Ildefonso Díaz \\ Department of Applied Mathematics, Facultad de Matemáticas, Universidad Complutense de Madrid, Plaza de las Ciencias, 3 , \\ 28040 Madrid, Spain
}

(Received 15 June 2007; published 19 September 2007)

\begin{abstract}
Global time-delay autosynchronization is known to control spatiotemporal turbulence in oscillatory reactiondiffusion systems. Here, we investigate the complex Ginzburg-Landau equation in the regime of spatiotemporal turbulence and study numerically how local or a combination of global and local time-delay autosynchronization can be used to suppress turbulence by inducing uniform oscillations. Numerical simulations show that while a purely local control is unsuitable to produce uniform oscillations, a mixed local and global control can be efficient and also able to create other patterns such as standing waves, amplitude death, or traveling waves.

DOI: 10.1103/PhysRevE.76.036209

PACS number(s): 82.40.Bj, 05.45.Gg
\end{abstract}

\section{INTRODUCTION}

Nonlinear dissipative media, ubiquitously found among biological, chemical, and physical systems, not only show temporally periodic behavior or give rise to regular, spatiotemporal patterns, but also display spatiotemporally disordered, chaotic states [1]. In the context of reaction-diffusion systems, in particular those chaotic states are of interest which are created due to the impact of spatial coupling-i.e., those which are induced by diffusion. It is a challenge to control such chaotic states and replace them by a simpler, regular behavior. It has been successfully demonstrated that reaction-diffusion systems can be controlled and engineered by external ("forcing") and internal ("feedback") signals [2].

In particular, feeding back a global, time-delayed signal to the system is a suitable way of controlling spatiotemporal chaos. This method was proposed in 1992 by Pyragas for dynamical systems described by ordinary differential equations and is called time-delay autosynchronization (TDAS) [3]. The main idea of TDAS is to apply to the system a signal $F$ which is proportional to the difference of the actual state of the system $A(t)$ at a given time $t$ with the state of the system $A(t-\tau)$ to the time $t-\tau$-i.e., $F(t, \tau) \propto A(t-\tau)-A(t)$. Timedelay autosynchronization has attracted much attention and numerous studies have applied, validated, and extended the original scheme (e.g., [4-8]). Its efficiency, in both numeric and experimental studies, has been shown for a wide range of systems (e.g., [9-13]). As the term autosynchronization indicates, the control force guides the system toward a previously unstable state where the feedback term finally vanishes, constituting a kind of noninvasive control. However,

\footnotetext{
*stichm@inta.es
}

the application of TDAS schemes does not necessarily lead to states where the feedback term vanishes. Examples of this kind of invasive feedback, in both experiment and theory, have been observed $[10,11]$ and will also be found here.

Aiming at controlling chaos in spatially-extended systems, two types of TDAS can be distinguished: First, global control, where the quantity that is fed back to the system is not space dependent-e.g., a global quantity or a spatial average of a space-dependent one. Second, local control, where the feedback variable depends on the spatial coordinate. There are many possibilities to realize local feedback schemes, and there have been (often motivated by some particular system) described methods involving spatial filtering [12] or spatially shifted domains $[14,15]$. In this paper, we focus on a completely local scheme where $A(x, t)$ is compared to $A(x, t-\tau)$ for all $x$. The majority of studies concentrate on global TDAS because it is the only possible realization for non-spatially-extended systems and because its implementation in real systems (even in those with spacedependent dynamics) is often easier than for local TDAS.

Two particularly interesting reaction-diffusion systems displaying spatiotemporal chaos and offering access for control are the photosensitive Belousov-Zhabotinsky reaction and the $\mathrm{CO}$ oxidation reaction on $\mathrm{Pt}(110)$ [2]. Nevertheless, our work is not performed in the setting of a particular reaction-diffusion system, but uses as framework the complex Ginzburg-Landau equation (CGLE), one of the most well-studied models of pattern-forming systems [16]. In the context of reaction-diffusion systems, the CGLE describes a spatially-extended system close to a supercritical Hopf bifurcation $[17,18]$. There, the system is governed by uniform, harmonic small-amplitude oscillations. However, in a certain parameter regime (if the Benjamin-Feir criterion is met), these oscillations are unstable and spatiotemporal chaos, also 
called turbulence, is observed. Two main types of spatiotemporal chaos can be distinguished: first, phase turbulence, where the amplitude of the oscillations varies only a little while the phases fluctuate and, second, defect or amplitude turbulence, where both amplitude and phase fluctuate strongly and the system dynamics is governed by defects, where the amplitude drops to zero. These kinds of turbulence are diffusion induced-i.e., cannot be found if diffusive, local coupling in space is suppressed.

In spite of the widespread use of the CGLE, investigations of TDAS in the context of the CGLE are relatively few. In the following, we mention shortly some publications relevant in this context: Bleich and Socolar studied the control of spatiotemporal chaos by traveling waves for the CGLE without global terms (but in the extended TDAS scheme with multiple delay times) [19]. Harrington and Socolar investigated the corresponding two-dimensional case [20]. Montgomery and Silber studied the CGLE with a local TDAS scheme where also spatially shifted terms of the form $A(x-\Delta x, t)$ were involved, focusing on the stabilization of traveling waves [15]. Recent work has investigated the dynamics of the same model in two space dimensions [21]. Based on previous work on the CGLE under the influence of global feedback $[22,23]$ and on TDAS in the CO oxidation reaction [11], Beta and Mikhailov investigated global TDAS in the CGLE, focusing on the question why for global TDAS noninvasive stabilization of uniform oscillations is not possible [10]. Boccaletti and Bragard managed to control turbulence in the CGLE by applying a control term involving a desired target state, however without delay [24]. Ramana Reddy et al. studied time-delay effects on coupled oscillators [25]. To our knowledge, a direct comparison of local and global feedback schemes for the CGLE has not been made. However, Beck et al. [26] and then Unkelbach et al. [27] compared different local and global control schemes for various semiconductor models.

The present work is motivated by the interest to understand the interplay of global and local TDAS in the standard CGLE. The article is organized as follows: in Sec. II, we introduce the CGLE with local and global control terms as basic model. In Sec. III, we numerically compute control diagrams and discuss the developing spatiotemporal solutions. Finally, we close the article with a discussion of the results (Sec. IV).

\section{COMPLEX GINZBURG-LANDAU EQUATION WITH FEEDBACK}

Reaction-diffusion systems can display various types of oscillatory dynamics. However, close to a supercritical Hopf bifurcation, all such systems are described by the complex Ginzburg-Landau equation [16-18], given by

$$
\partial_{t} A=(1-i \omega) A-(1+i \alpha)|A|^{2} A+(1+i \beta) \nabla^{2} A,
$$

where $A$ is the complex oscillation amplitude, $\omega$ the linear frequency parameter, $\alpha$ the nonlinear frequency parameter, $\beta$ the linear dispersion coefficient, and $\nabla^{2}$ the Laplacian operator.

For a one-dimensional system, the CGLE with time-delay autosynchronization reads

$$
\begin{gathered}
\partial_{t} A=(1-i \omega) A-(1+i \alpha)|A|^{2} A+(1+i \beta) \partial_{x x} A+F, \\
F=\mu e^{i \xi}\left\{m_{l}[A(x, t-\tau)-A(x, t)]+m_{g}[\bar{A}(t-\tau)-\bar{A}(t)]\right\},
\end{gathered}
$$

where

$$
\bar{A}(t)=\frac{1}{L} \int_{L} A(x, t) d x
$$

denotes the spatial average of $A$ over a one-dimensional medium of length $L$. The parameter $\mu$ describes the feedback strength, and $\xi$ characterizes a phase shift between the feedback and the dynamics. The parameters $m_{g}$ and $m_{l}$ denote the global and local feedback contributions, respectively. The feedback term can be split into global and local parts

$$
\begin{aligned}
F=F_{l}+F_{g}= & m_{l} \mu e^{i \xi}[A(x, t-\tau)-A(x, t)] \\
& +m_{g} \mu e^{i \xi}[\bar{A}(t-\tau)-\bar{A}(t)] .
\end{aligned}
$$

If $m_{l}=0$, the case of global TDAS, studied by Beta and Mikhailov [10], is retrieved.

If we look for solutions of uniform oscillations $A(t)$ $=\rho_{0} \exp (-i \Omega t)$, we obtain for the feedback term

$$
F=A \mu\left(m_{g}+m_{l}\right)\left(e^{i(\xi+\Omega \tau)}-e^{i \xi}\right)
$$

and for the amplitude and frequency of uniform oscillations

$$
\begin{gathered}
\rho_{0}=\sqrt{1+\mu\left(m_{g}+m_{l}\right) \chi_{1}}, \\
\Omega=\omega+\alpha+\mu\left(m_{g}+m_{l}\right)\left(\alpha \chi_{1}-\chi_{2}\right),
\end{gathered}
$$

where $\chi_{1,2}$ denote effective modulation factors, which can be positive or negative and which arise from the feedback and hence depend on $\xi$ and $\tau$ :

$$
\begin{aligned}
& \chi_{1}=\cos (\xi+\Omega \tau)-\cos \xi, \\
& \chi_{2}=\sin (\xi+\Omega \tau)-\sin \xi .
\end{aligned}
$$

Since $\chi_{1,2}$ also depend on $\Omega$, no explicit analytic solutions for Eqs. (6a) and (6b) can be given. In the absence of feedback, the period $T_{0}$ of uniform oscillations with amplitude $\rho_{0}=1$ is $T_{0}=2 \pi / \Omega=2 \pi /(\omega+\alpha)$. We assume that the Benjamin-Feir criterion is $1+\alpha \beta<0$ fulfilled-i.e., that these uniform oscillations are unstable.

\section{NUMERICAL SIMULATIONS}

In this section, we present the results of a numerical study of Eqs. (2a) and (2b). First, we give an overview of the different patterns that are found for different TDAS schemes. Then, we study more in detail the transitions from one pattern to another for mixed TDAS.

For time integration, we use an explicit Euler scheme with $\Delta t=0.002(\Delta t=0.001$ for single simulations to assure convergence). The Laplacian operator is discretized using a nextneighbor representation. The system size of the onedimensional medium is $L=128$ with a spatial resolution of $\Delta x=0.32$. We apply periodic boundary conditions, and 
(a)

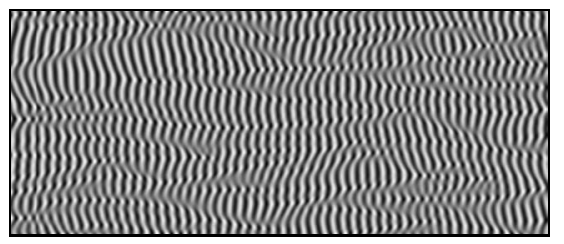

(b)

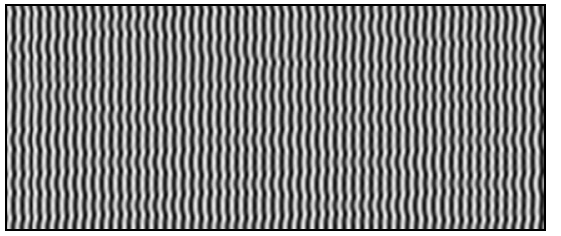

(c)

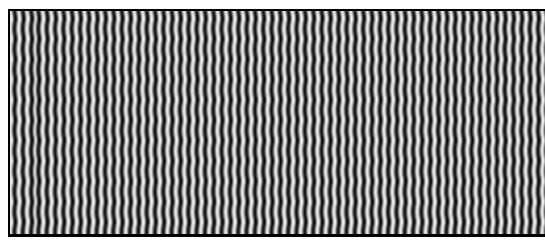

(d)

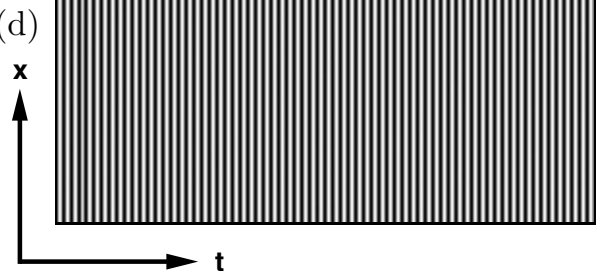

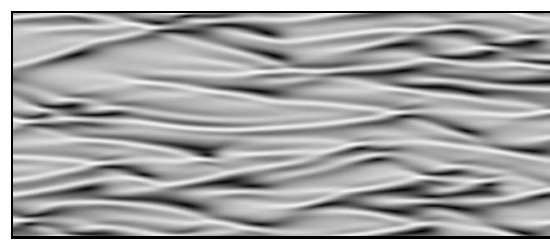
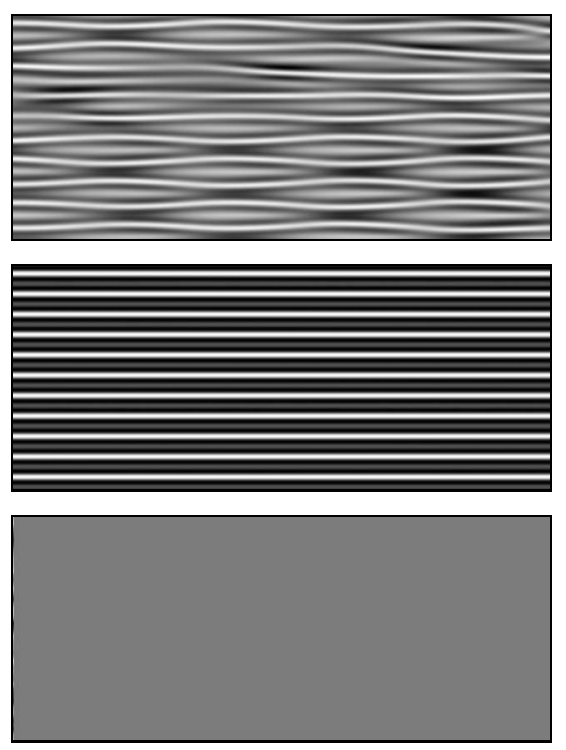

FIG. 1. Main spatiotemporal solutions for different feedback magnitudes. Shown are spacetime diagrams in gray scale for $\operatorname{Re} A$ (left panel) and $|A|$ (right panel) for a time interval of $t=50$ in the asymptotic regime. Throughout the article, black (white) denotes low (high) values of the respective quantity (rescaled for each simulation). The parameters are $m_{l}=1.4, m_{g}=0.6$, and $\tau=0.3$. The values of $\mu$ are $\mu=0.05$ (a), $\mu=0.23$ (b), $\mu=0.35$ (c), and $\mu=0.50$ (d). The other parameters are $\alpha=-1.4, \beta=2, \omega$ $=2 \pi-\alpha$, and $\xi=\pi / 2$. the initial conditions consist of either slightly perturbed uniform oscillations or developed spatiotemporal chaos. For $t \in[-\tau, 0]$, variables are zero. The overall simulation time for a given parameter set is $t=700$ (usually the systems reach the stable asymptotic state before $t=200$ ). To scan the parameter space $(\tau, \mu), \tau$ was changed in steps of 0.05 from 0.05 to 2.0 and $\mu$ in steps of 0.05 from 0.05 to 1.0 . We do not discuss values of $\mu>1$ since then the amplitude of the control term can become much larger than the amplitude of the uncontrolled limit cycle with amplitude of order 1 . For the same reason, we keep $m_{l}+m_{g}=2$ constant. The parameter $\xi$ is not varied systematically from the value given below, although we performed some rough parameter scans for $\xi=\pi$ and $\xi$ $=1.25 \pi$, which, however, do not reveal qualitatively novel behavior.

For the sake of comparison with Ref. [10], the parameters in this section are chosen as $\alpha=-1.4, \beta=2, \omega=2 \pi-\alpha$ $\approx 7.68$, and $\xi=\pi / 2$. Therefore, the Benjamin-Feir criterion $1+\alpha \beta<0$ is met and we observe amplitude turbulence in the absence of feedback.

\section{A. Pattern overview}

In this section, we first show examples of the most important spatiotemporal patterns. Then, we plot the stability diagrams of these solutions in parameter space. We start with a case of mixed global and local TDAS where the global parts clearly dominate. Then, we increase the contribution of the local TDAS term and finish with the case of purely local TDAS. Finally, we show examples of interesting, but not so common patterns.
In Fig. 1, we see the most important spatiotemporal patterns that are observed for combined local and global TDAS. For low feedback magnitude [Fig. 1(a)], amplitude turbulence similar to the one found without feedback is observed. The wave field fluctuates irregularly, and the modulus of the amplitude $|A|$ drops down to zero frequently [dark black areas in right panel of Fig. 1(a)]. For larger values of $\mu$, the medium still shows irregular dynamics, however with obvious similarity to standing waves [Fig. 1(b)]. In analogy to the phase turbulence for the standard CGLE, this pattern can be denoted as phase-turbulent standing waves. In this example, the amplitude oscillates in both space and time between the values of 0.45 and 1 , however without dropping to zero. The change of a completely chaotic state known as amplitude turbulence to an almost regular state is smooth. A detailed characterization of the different turbulent states is beyond the scope of this article.

For intermediate feedback strengths, the oscillations regularize and standing waves form as spatial modulations of uniform oscillations [Fig. 1(c)]. The modulus of the amplitude $|A|$ oscillates in space between 0.68 and 0.88 and remains temporally constant. Finally, for large feedback values [Fig. 1(d)] oscillations are uniform, with $|A| \approx 0.75$ in this case.

To get an overview of the stable spatiotemporal solutions of the system, we vary the delay time $\tau$, the feedback magnitude $\mu$, and the contributions of local and global feedback. In Fig. 2, we show diagrams of the solutions in the parameter space spanned by $\tau$ and $\mu$.

First, we discuss the case when local and global feedback terms contribute with the same strength $m_{l}=m_{g}=1$ [Fig. 2(a)]. For very low $\mu$, no control is possible and spatiotem- 
(a)

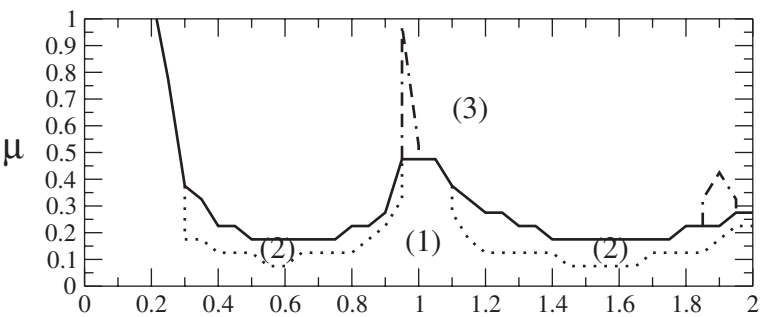

(b)

(c)

(d)

(e)
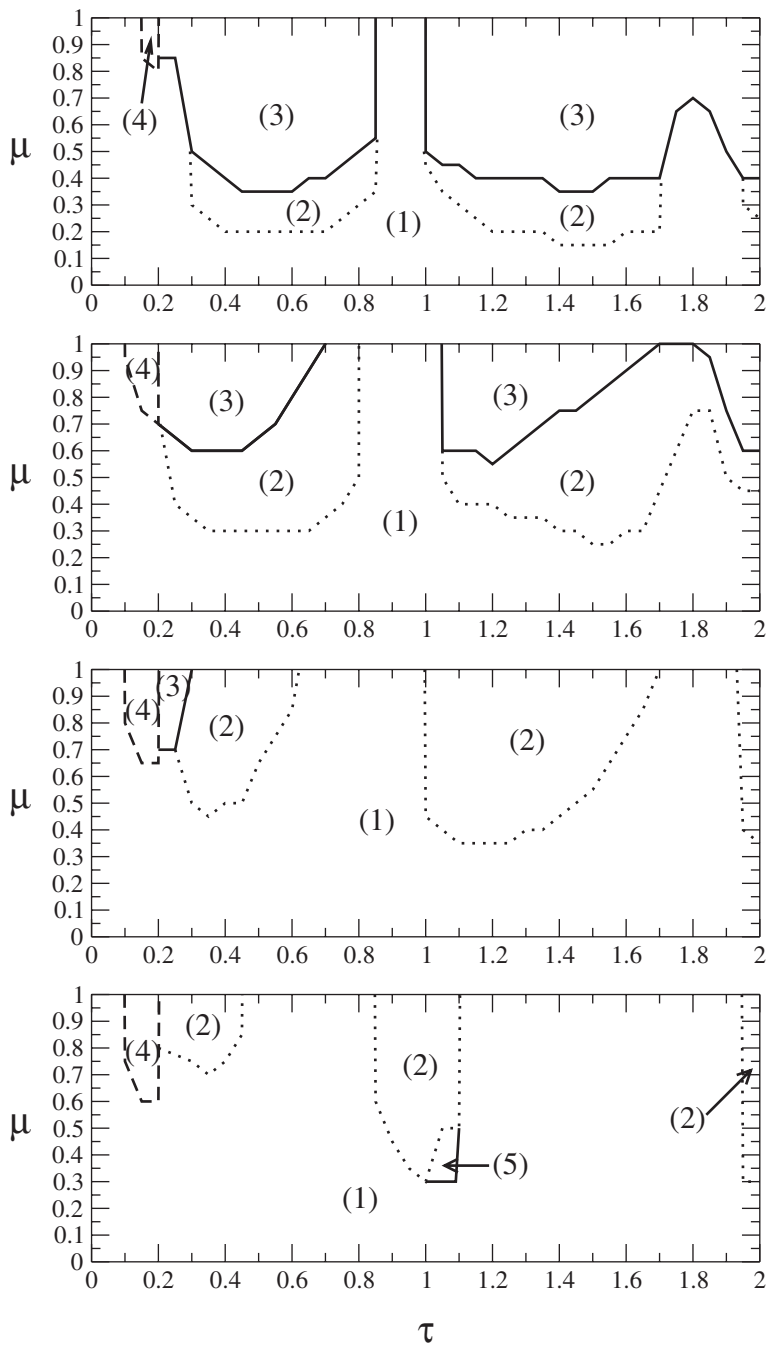

FIG. 2. Diagram of spatiotemporal solutions. The parameters are $m_{g}=1, m_{l}=1$ (a), $m_{g}=0.6, m_{l}=1.4$ (b), $m_{g}=0.4, m_{l}=1.6$ (c), $m_{g}$ $=0.2, m_{l}=1.8(\mathrm{~d})$, and $m_{g}=0, m_{l}=2(\mathrm{e})$. The other parameters are as in Fig. 1. The numbers denote following regimes: spatiotemporal chaos (1), standing waves (2), uniform oscillations (3), amplitude death (4), and traveling waves (5). Part (a) is obtained from simulations with two initial conditions, (b)-(e) only from initial conditions representing developed chaos. For more information, see the main text.

poral chaos [denoted as (1) throughout Fig. 2] is always observed, independently of the value of $\tau$. Also, for small $\tau$, no control is achieved since the difference between the actual and time-delayed values (of either global or local $A$ ) is too small to produce an efficient control signal.

For wide ranges of $\tau$, chaos can be suppressed already for relatively small feedback magnitudes. Then, standing waves appear, denoted as (2). As $\mu$ is increased further, the amplitude of the standing waves decreases until finally uniform oscillations (3) are formed. In order to keep the diagram simple, only perfectly regular standing waves are denoted as (2); the phase-turbulent standing waves shown in Fig. 1(b) belong still to the turbulent regime denoted as (1).

Figure 2(a) was created using runs with two different initial conditions: First, slightly perturbed uniform oscillations and, second, developed chaos. The simulations show hysteresis between turbulence and uniform oscillations. In the triangular-shaped regions for values of $\tau$ close to multiple values of the period of oscillations $\tau \approx n T_{0}, n=1,2\left[T_{0}\right.$ $=2 \pi / \Omega=2 \pi /(\omega+\alpha)=1]$, either uniform oscillations or turbulence can be found. Only in Fig. 2(a) do we display the hysteresis, and Figs. 2(b)-2(e) are obtained with runs starting with developed chaos as initial condition, in order to focus on the issue of controlling turbulence.

The shape and location of the pattern regimes in parameter space of Fig. 2(a) are similar to the case without local TDAS, presented in Fig. 9 of Ref. [10], where also hysteresis and complex patterns were already reported. Because of this similarity, we conclude that the effect of the local feedback compared to the global one is small and that we have to go to larger values of $m_{l}$ if we want to explore the specific impact of local TDAS on the dynamics.

In Fig. 2(b) we show the parameter space with a stronger contribution of local feedback, $m_{l}=1.4$ and $m_{g}=0.6$. Since we want to avoid large amplitudes and therefore large contributions from both feedback terms at the same time, we decrease $m_{g}$ as we increase $m_{l}$. Comparing with Fig. 2(a), several features in Fig. 2(b) catch our attention. First, larger values of $\mu$ are needed in order to induce both standing waves and uniform oscillations. In particular, turbulence is already difficult to control for delay times close (and slightly smaller than) multiples of the period of oscillations without feedback. Second, the regions where standing waves are stabilized are significantly larger than for smaller $m_{l}$ (and larger $m_{g}$ ). Third, for small values of $\tau \approx 0.2$ and large feedback values $\mu>0.85$, amplitude death is observed. This means that instead of uniform oscillations, a stationary state is stabilized. Simulations not shown here show that amplitude death can be induced for large global feedback magnitudes (e.g., $\mu=13$ ) also for $m_{l}=0$.

As the weight of the local feedback term is further increased $\left[m_{g}=0.4\right.$ and $m_{l}=1.6$, Fig. $\left.2(\mathrm{c})\right]$, the stabilization of standing waves and uniform oscillations becomes more and more difficult and the limiting curves shift up. Still, the region where stable standing waves are found expands at the cost of uniform oscillations. Also, the area in parameter space where amplitude death occurs becomes larger.

Changing the parameters from $m_{g}=0.4$ and $m_{l}=1.6$ [Fig. $2(\mathrm{c})]$ to $m_{g}=0.2$ and $m_{l}=1.8$ [Fig. $2(\mathrm{~d})$ ], a relatively strong shift of the curves in parameter space is observed. Within the considered values of $\mu$, stabilization of uniform oscillations is now limited to a small region close to the area of amplitude death. Although there are regions where standing waves are stable, in a large part of the studied parameter space actually no control of any regular pattern is observed and turbulent states persist (at least during the simulation time used). These turbulent states do not necessarily resemble am- 
(a)

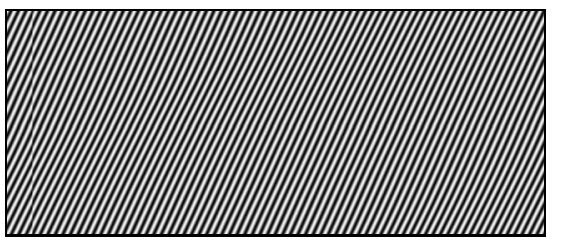

(b)

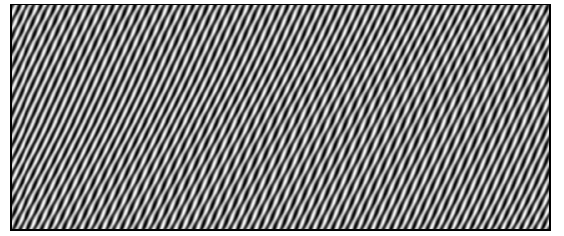

(c)

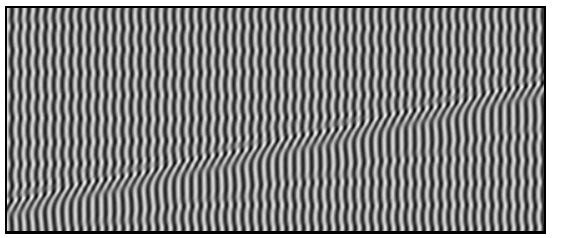

(d)

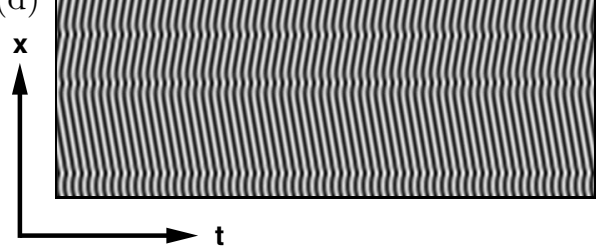

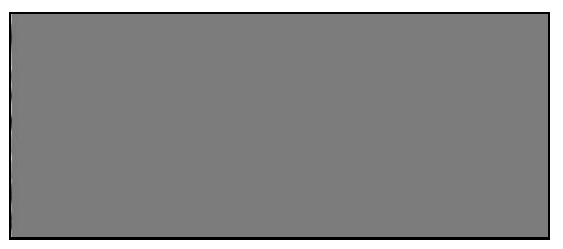
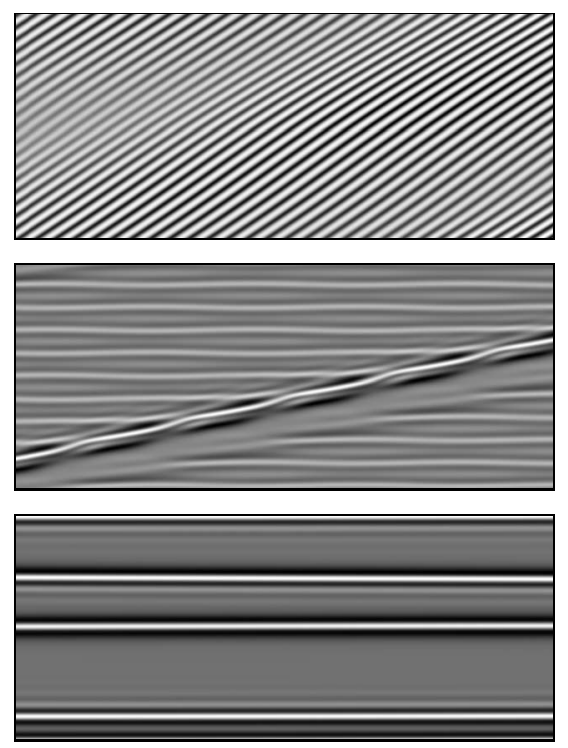

FIG. 3. Other spatiotemporal solutions. Shown are space-time diagrams in gray scale for $\operatorname{Re} A$ (left panel) and $|A|$ (right panel) for a time interval of $t=50$. The parameters are $m_{l}=2.0, m_{g}=0, \tau$ $=1.05, \mu=0.30$ (a), $m_{l}=2.0, m_{g}$ $=0, \tau=1.05, \mu=0.45$ (b), $m_{l}=1.6$, $m_{g}=0.4, \tau=1.1, \mu=0.3$ (c), and $m_{l}=2.0, m_{g}=0, \tau=1.05, \mu=0.45$ (d). The minimum (maximum) values of $|A|$ for (a) are 0.77 (0.77), for (b) 0.60 (1.08), for (c) 0.43 (1.10), and for (d) 0.66 (1.00). The other parameters are as in Fig. 1. plitude turbulence for $\mu=0$, since the feedback terms always act on the dynamics.

Finally, the case of completely local TDAS $\left(m_{g}=0\right.$ and $\left.m_{l}=2\right)$ is presented in Fig. 2(e). The simulations show that for completely local TDAS transients are longer, initial conditions influence considerably the transient and asymptotic dynamics, and hysteresis for the patterns is present. Therefore, Fig. 2(e) can only roughly describe the behavior of the system for vanishing global TDAS. The main results of our simulations are that uniform oscillations are not found (for the parameters used), the region of amplitude death is enlarged, standing waves are present but mostly irregular, and traveling waves appear.

For the values of $\mu$ that we used $(\mu \leq 2)$, we do not find stabilization of uniform oscillations. Compared to Fig. 2(c), the region of amplitude death is enlarged and the curves limiting the standing waves move toward larger values of $\mu$. However, for vanishing global contribution the standing waves that we have found seem to be mostly irregular. In contrast to Figs. 2(a)-2(d) the regions denoted as (2) in Fig. 2(e) therefore also include irregular standing waves. Still, single simulations not shown here demonstrate that regular standing waves created for $m_{g} \neq 0$ can remain stable with respect to small perturbations for $m_{g}=0$ (at least for times up to $10^{2}$ ). Therefore, global TDAS is not essential to have stable regular standing waves, although favorable.

As a novel feature, however, stable traveling waves are found in a small region for $\tau \approx 1$ and $\mu \approx 0.4$, adjacent to the region of standing waves. Two types of traveling waves are found. For relatively small $\mu$ and $\tau$, traveling waves can be perfectly harmonic and have a spatially constant amplitude $|A|$. However, many traveling waves, and in particular those with large $\mu$ and $\tau$, show a spatially periodic modulation in $|A|$, similar to the amplitude modulation representing standing waves. Furthermore, traveling waves are also seen in a irregular fashion-i.e., without a perfect symmetry-again similar to irregular standing waves. In Fig. 3(a), we display a traveling wave with constant $|A|$ and in Fig. 3(b) a traveling wave with amplitude modulation. Simulations not shown here reveal that a traveling-wave state created for $m_{g}=0$ can survive-although distorted and irregular-small global TDAS terms $\left(m_{g}=0.1\right.$ and $m_{l}=1.9$ as an example).

In many simulations we have also seen more complex patterns such as traveling localized structures, asymmetric standing waves, or low-amplitude, long-wavelength patterns. These patterns are sensitive to initial conditions and are often found in small parameter regions between two distinct main pattern regimes. Although we do not focus on these patterns, we show as examples a localized traveling structure [Fig. 3(c)] and a state of a four localized structures [Fig. 3(d)]. Localized traveling structures have been found for many simulations and can be created by perturbing locally a standing-wave pattern. Figure 3(c) shows a simulation where a localized structure, corresponding to a phase flip of $2 \pi$ (or kink), travels on the background of slightly irregular standing waves. The simulation presented in Fig. 3(d)-for the same parameters as in Fig. 3(b), but with different initial conditions - shows a medium where the waves in the upper half travel in an opposite direction than the waves in the lower half. Therefore, the localized structure in the center of the medium can be interpreted as a pacemaker. Within the upper half, a phase flip is found that represents a perturbation of the wave field. At the upper boundary and close to the lower boundary, two other localized structures are found that represent wave sinks. Since we use periodic boundary con- 


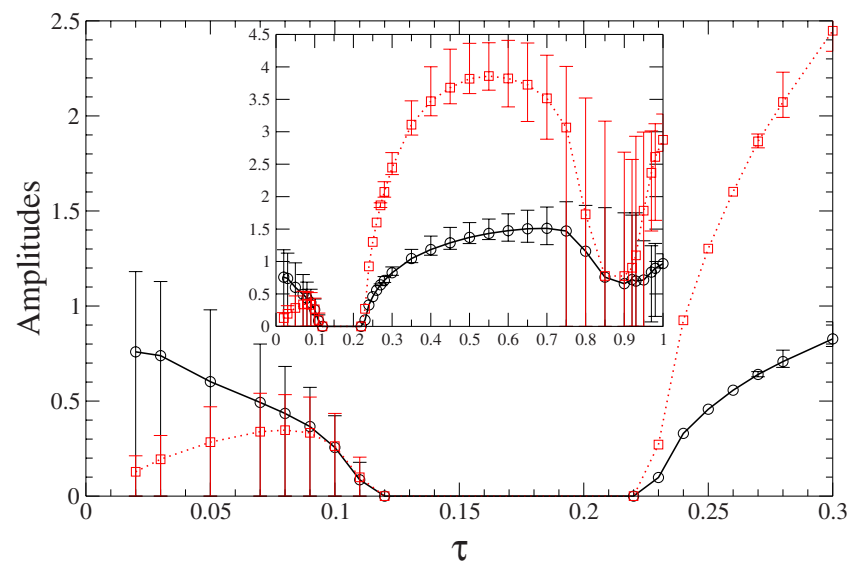

FIG. 4. (Color online) Average and extremal values of $|A|$ and $|F|$. The parameters are $m_{g}=0.2, m_{l}=1.8$, and $\mu=0.75$. The other parameters are as in Fig. 1. The main figure shows $\tau$ from 0 to 0.3 ; the inset shows $\tau$ from 0 to 1 . The black solid line shows $|A|$, the red dotted line $|F|$. The bars denote the maximum and minimum values of the magnitudes in a time interval $t=50$.

ditions, these structures are actually located close to each other and can be regarded as a bound state of localized structures. Since in this simulation the localized structures move slowly, the asymptotic state is not yet reached. Nevertheless, we can speculate that in the absence of a phase flip, the pacemaker could be stable.

\section{B. Pattern transitions for mixed TDAS}

If we want to understand how a stable pattern is replaced by another one as we change parameters, it is useful to characterize the patterns in terms of the minimum, average and maximum values of the modulus of the oscillation amplitude $|A|$ and the feedback amplitude $|F|$ during a given time interval. The average value (and accordingly minimum and maximum values) are determined as an average over the system size $L=128$ and over a time interval $t=50$ in the asymptotic regime.

As an example, we fix $\mu=0.75$ and vary $\tau$ from 0 to 1 for the case $m_{g}=0.2$ and $m_{l}=1.8$ [Fig. $2(\mathrm{~d})$ ]. The minimum, average, and maximum values of the modulus of the oscillation amplitude $|A|$ and the feedback amplitude $|F|$ for the different patterns are shown in Fig. 4.

Since in the limit $\tau \rightarrow 0$ the feedback terms vanish, it is not surprising that for small $\tau,|A|$ takes values close to 1 and $|F|$ takes values close to 0 . As $\tau$ increases, $|A|$ decreases and $|F|$ increases until around $\tau \approx 0.09$ the amplitudes are of the same magnitude. Then, the magnitudes $|A|$ and $|F|$ basically coincide (even if no spatial average is performed) and the curve for $|F|$ begins to coincide with the decreasing curve of $|A|$. For $\tau \approx 0.12$, both amplitudes vanish and amplitude death is observed. Since $|F|=0$, control of the steady state is noninvasive. Note that for values $\tau<0.12$, spatiotemporal chaos is observed, reflected by the fact that the minimum values of $|A|$ and $|F|$ are 0 .

Amplitude death is found up to $\tau \approx 0.22$, when uniform oscillations appear which can be clearly identified since

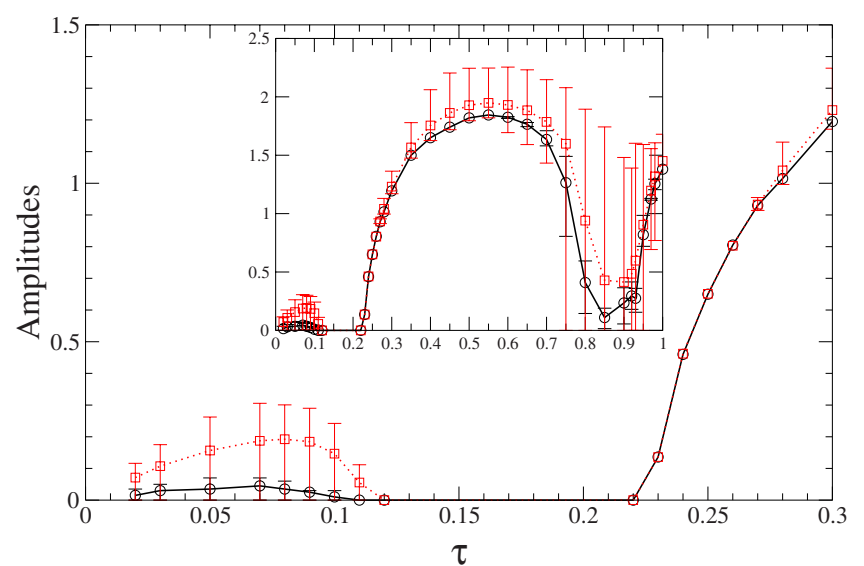

FIG. 5. (Color online) Average and extremal values of local $\left|F_{l}\right|$ and global $\left|F_{g}\right|$ contributions of $F$. The parameters are as in Fig. 4. The main figure shows $\tau$ from 0 to 0.3 ; the inset shows $\tau$ from 0 to 1. The black solid line shows $\left|F_{g}\right|$, the red dotted line the local contribution $\left|F_{l}\right|$. Both curves have been rescaled with their magnitude parameter-i.e., $\left|F_{l}\right| / m_{l}$ and $\left|F_{g}\right| / m_{g}$. The bars denote the maximum and minimum values of the magnitudes in a time interval $t=50$.

maximum, average, and minimum values of $|A|$ (and those of $|F|$ ) coincide. For all $\tau>0.22,|F|$ never drops to zero and usually is much larger than $|A|$. From uniform oscillations, we enter the regime of standing waves if $\tau$ increases further. The standing-wave pattern develops softly from uniform oscillations-i.e., starts with a small, then increasing amplitude.

For this parameter set, the region where standing waves are observed is large (from $\tau \approx 0.27$ up to $\tau \approx 0.7$, as seen in the inset of Fig. 4). The modulus of the amplitude increases up to values $|A| \approx 1.5$ and also the amplitude of the standingwave pattern, reflected by the increase of maximum and minimum values of $|A|$. In this regime, the feedback amplitude $|F|$ is very large, reaching $|F| \approx 4$, and it decreases slightly toward the upper end of the range of standing waves. To be precise, for $\tau \geq 0.35$, the standing waves show in their amplitude profile in space a period- 2 behavior. As $\tau$ increases, the additional small peaks increase in magnitude until the pattern starts to breathe around $\tau \approx 0.5$ where $|F|$ reaches its maximum. Hence, for $0.5 \leq \tau \leq 0.7$ breathing standing waves are seen. Breathing becomes increasingly irregular toward larger values of $\tau$.

As $\tau$ is increased further, $\tau \geq 0.75$, standing waves are replaced by spatiotemporal chaos. Unlike in the regime for small $\tau$, here $|F|>|A|$, so hence the feedback always has a large impact on the dynamics. The modulus of the amplitude $|A|$ decreases from values of about 1.5 to 0.75 and then starts to rise again. For values $\tau \approx 1$, standing waves are recovered. No reappearance of amplitude death or uniform oscillations are observed, although it seems possible that these patterns may be found for $\mu>1$.

To clarify the relative influence of the different feedback terms, we display in Fig. 5 the curves of the average values of $\left|F_{l}\right|$ and $\left|F_{g}\right|$. Since the strength of the two feedbacks is different, $m_{l}=1.8$ vs $m_{g}=0.2,\left|F_{l}\right|>\left|F_{g}\right|$ practically always and we rescale the feedback amplitudes by the values of the 


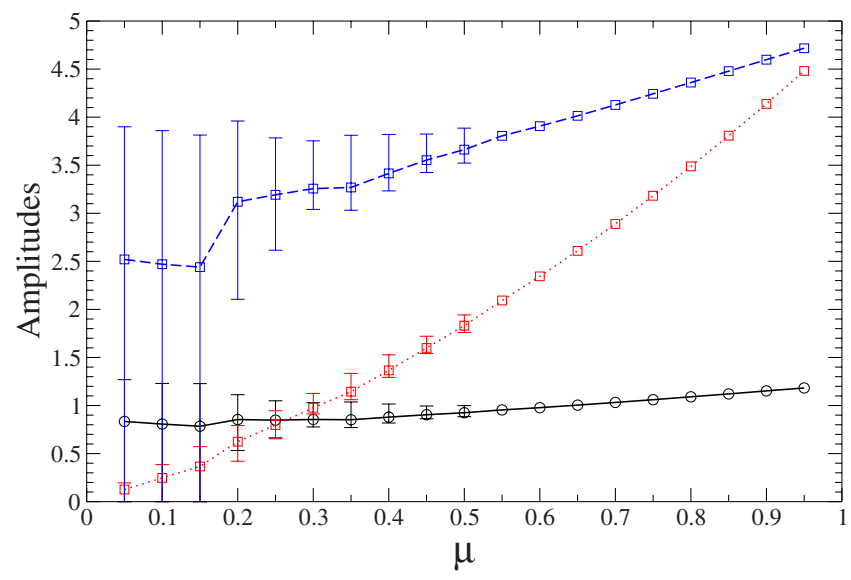

FIG. 6. (Color online) Average and extremal values of $|A|$ and $|F|$. The parameters are $m_{g}=0.4, m_{l}=1.6$, and $\tau=0.35$. The other parameters are as in Fig. 1. The black solid line shows $|A|$, the red dotted line $|F|$, and the blue dashed line $|F| / \mu$. The error bars denote the maximum and minimum values of the magnitudes in a time interval $t=50$.

feedback strength-i.e., $\left|F_{l}\right| / m_{l}$ and $\left|F_{g}\right| / m_{g}$ - to compare the relative influence of the different contributions. We observe that in the chaotic regime the local feedback contribution dominates clearly. This is due to the fact that for chaotic dynamics the spatial average of a variable yields small values while the local terms are large. In particular, as the amplitude of the chaotic oscillations decreases as the regime of amplitude death is approached, the local contribution is much larger than the global one, indicating that the local feedback terms are important to observe amplitude death. In the regime of uniform oscillations, the two feedback terms give the same contribution, as can be expected since uniform oscillations are by definition non-space-dependent. As the onset of standing waves is crossed, the impact of the local feedback again starts to dominate over the global feedback. The contribution of the local feedback becomes increasingly larger. Therefore, the presence of mixed local and global contributions seems to favor standing-wave patterns. This is in agreement with the fact that standing waves are found in larger regions of parameter space as the local feedback term enters more strongly. As we go to larger values of $\tau$, where again spatiotemporal chaos is observed, local contributions remain stronger than global ones.

In Fig. 6, $|A|$ and $|F|$ are plotted for $m_{g}=0.4, m_{l}=1.6, \tau$ $=0.35$, and varying $\mu$. For this set of parameters, we observe three main regimes: spatiotemporal chaos, standing waves, and uniform oscillations [cf. Fig. 2(c)]. In the limit $\mu \rightarrow 0$, we recover the native amplitude turbulence present in the case without feedback. As the feedback strength $\mu$ increases, also $|F|$ increases and $|A|$ decreases slightly. Then, at $\mu$ $=0.20$, the mean value of $|A|$ jumps to a larger value. Since the minimum values of $|A|$ and $|F|$ do not drop to zero, it becomes clear that we have left the region of amplitude turbulence. Actually, for the values $\mu=0.20$ and $\mu=0.25$, the simulations show irregular and oscillating standing-wave patterns.

Since $F \propto \mu$ [Eqs. (2a) and (2b)], it is not surprising to observe a nearly linear increase of $|F|$ with $\mu$ for the whole range of $\mu$. Therefore, it is interesting to display $|F| / \mu$ to reveal changes of $|F|$ which are not due to the direct, linear contribution of $\mu$. This curve shows a strong shift at $\mu$ $=0.2$ as we go from spatiotemporal chaos to irregular standing waves. Although these standing waves are very irregular and also represent a state of spatiotemporal chaos, there is a clear difference between the regimes; e.g., the amplitude $|A|$ does not drop to zero anymore. Regular standing waves are found between $0.3 \leq \mu \leq 0.5$. The standing waves become regular at a value for $\mu$ where the modulus of the feedback overcomes the modulus of the amplitude, $|F|>|A|$.

As $\mu$ is increased within the regime of standing waves, their amplitude decreases and approaches zero as the regime of uniform oscillations are reached at $\mu \approx 0.55$. All displayed amplitudes increase constantly within this regime, and in particular $|F|$ reaches values larger than 4 for $\mu>0.9$. The curve for $|F| / \mu$ increases almost over the whole range of $\mu$ and thus reflects the fact that $|F|$ increases stronger than linearly.

\section{DISCUSSION}

In this article, we have investigated the behavior of an oscillatory medium described by the complex GinzburgLandau equation influenced by local and global feedback terms actuating through a TDAS scheme. Before discussing our findings in light of other publications, we shortly summarize the results.

In Figs. 2-6 we have presented numerical results for TDAS feedback with local and global contributions. In Fig. 2 we have given an overview of the spatiotemporal solutions in parameter space $(\tau, \mu)$ for different combinations of local and global TDAS terms. We have displayed several quantities (modulus of the total, local, and global feedback terms) which characterize and reflect the changes between different spatiotemporal patterns as $\tau$ (Figs. 4 and 5) and $\mu$ (Fig. 6) are varied. Particularly, important patterns beside uniform oscillations are standing waves, traveling waves, amplitude death, and different kinds of spatiotemporal chaos, such as phaseturbulent standing waves (Figs. 1 and 3). Also, $2 \pi$-phase flips and pacemakers have been observed (Fig. 3).

If global TDAS is present, small local TDAS terms have only little impact on the dynamics and pattern formation is similar to the system studied by Beta and Mikhailov [10]. As local TDAS control terms become stronger, the regions in parameter space where uniform oscillations are found shrink and the areas where chaos and standing waves predominate expand. Standing-wave patterns are also observed in breathing and slightly irregular variants, being the latter particularly important for pure local TDAS. We conclude that local feedback terms make the stabilization of uniform oscillations difficult and favor the formation of standing waves instead. Nevertheless, since also those regions become larger where spatiotemporal chaos persists, we have to state that not only the creation of spatially uniform, but also of spatially periodic regular solutions becomes increasingly difficult as local TDAS terms become more dominant. To observe stable, perfectly regular standing waves, a combination of local and global TDAS terms seems favorable. 
We are interested in the control of turbulent states and hence most simulations start with developed spatiotemporal chaos. We observe hysteresis effects, such as between uniform oscillations and turbulence and standing waves and turbulence, but since hysteresis between uniform oscillations and turbulence was already reported for purely global TDAS [10], we do not investigate this issue in detail.

Furthermore, we observe amplitude death. Typically, amplitude death has been studied for systems of coupled oscillators where the oscillators have a distribution of natural frequencies $[28,29]$. This dynamical phenomenon consists of the coordinated decay of oscillations in a network of coupled oscillators. Nevertheless, this pattern is more general and can also be induced, e.g., by time delay for an array of identical oscillators coupled globally [25]. Atay showed that distributed delays (rather than distributed frequencies) can also facilitate amplitude death for coupled oscillators [30]. Stabilization of steady states by means of TDAS has been studied by Hövel and Schöll [31] and it may be interesting to connect their findings for a two-dimensional ordinary differential equation system with the results for coupled oscillators and spatially-extended systems. Similar to the case of standing waves, also the region of amplitude death expands as the local TDAS contribution increases, although in a lesser extent. Only one "death island" is found, for small $\tau$, although it seems possible that for large $\mu$ and $\tau$ more death islands could be discovered. Although amplitude death is particularly visible for local TDAS, we have observed amplitude death also for mixed TDAS and global TDAS. Hence, although not strictly necessary, local TDAS terms seem to favor the occurrence of amplitude death. Besides, amplitude death is the only pattern stabilized noninvasively-i.e., for vanishing feedback magnitude. All other patterns have nonzero feedback magnitudes. For uniform oscillations and purely global TDAS, this has been shown to be due to the presence of other unstable modes which prevents the stabilization of the native, unstable solution [10].

For purely local TDAS, we have observed the creation of traveling waves. Such patterns were already reported and investigated for (slightly different) local TDAS coupling schemes before [15,19-21]. Although we have not studied traveling waves in detail, we can state that such patterns have not been observed to appear spontaneously if global TDAS terms are present. Nevertheless, if we start from a travelingwave state and allow small global TDAS terms, traveling waves persist (distorted and irregular, although perfectly recognizable) and decay only for considerable contributions of global TDAS terms. However, we should have in mind that for vanishing global contributions, transients become very long, dependence on initial conditions is strong, and the simulations presented here can only give us a rough view of the system dynamics.
The findings presented above may be interpreted in the following way: First, global TDAS tries to stabilize uniform oscillations, although standing waves are admitted stable solutions in a small parameter region. Second, if local TDAS prevents the formation of uniform oscillations, at least standing waves may be induced and stabilized. Indeed, standing waves seem the preferred pattern for mixed TDAS schemes. Third, for purely local TDAS, even standing waves become unstable and traveling waves are observed. Hence, standing waves can be interpreted as a "compromise pattern" where traveling waves (favored by local TDAS) and uniform oscillations (induced by global TDAS) compete.

We have also observed traveling localized patterns, identified as $2 \pi$-phase flips, and (at least as a transient) pacemakers. Given the complexity of the system studied, it is not too surprising to find also these spatiotemporal structures in this systems. Actually, these patterns are known to occur already for global coupling (e.g., [32,33]). Still, it demonstrates that TDAS may represent another possibility to induce selforganized pacemakers in reaction-diffusion systems, a topic studied intensively during the last decades (see, e.g., Refs. $[34,35]$ and references therein). Since the pacemaker appears as a localized pattern connecting different traveling wave states, we can expect pacemakers especially for vanishing (or small) contributions of the global TDAS term.

An explicit comparison of local and global control schemes has been presented by Beck et al. [26] and Unkelbach et al. [27] for different semiconductor models. In particular, Unkelbach et al. [27] show that an exclusive local control may be even superior than a global or combined control in order to stabilize oscillations. Since their models are quite different from the one studied here, our results do not contradict their findings, although they make clear that the efficiency of local versus global control may depend strongly on the nature of the system under study.

To summarize, we can state that the presence of local TDAS terms prevents the stabilization of uniform oscillations and favors the formation of other spatiotemporal patterns, which for purely global TDAS would be either impossible, unstable, or suppressed. To characterize these patterns, such as standing waves, traveling waves, amplitude death, phase flips, or pacemakers, in more detail for a combination of local and global TDAS is certainly an interesting subject for future investigations.

\section{ACKNOWLEDGMENTS}

M.S. acknowledges financial support of the EU and of INTA. The research of J.I.D. is partially supported by Project No. MTM2005-03463 by DGISGPI.
[1] A. S. Mikhailov and A. Y. Loskutov, Foundations of Synergetics II, 2nd ed. (Springer-Verlag, Berlin, 1996).

[2] A. S. Mikhailov and K. Showalter, Phys. Rep. 425, 79 (2006).

[3] K. Pyragas, Phys. Lett. A 170, 421 (1992).
[4] J. E. S. Socolar, D. W. Sukow, and D. J. Gauthier, Phys. Rev. E 50, 3245 (1994).

[5] N. Baba, A. Amann, E. Schöll, and W. Just, Phys. Rev. Lett. 89, 074101 (2002). 
[6] W. Just, S. Popovich, A. Amann, N. Baba, and E. Schöll, Phys. Rev. E 67, 026222 (2003).

[7] B. Fiedler, V. Flunkert, M. Georgi, P. Hövel, and E. Schöll, Phys. Rev. Lett. 98, 114101 (2007).

[8] A. C. Casal and J. I. Díaz, Math. Models Meth. Appl. Sci. 16, 1 (2006).

[9] S. Schikora, P. Hövel, H.-J. Wünsche, E. Schöll, and F. Henneberger, Phys. Rev. Lett. 97, 213902 (2006).

[10] C. Beta and A. S. Mikhailov, Physica D 199, 173 (2004).

[11] C. Beta, M. Bertram, A. S. Mikhailov, H. H. Rotermund, and G. Ertl, Phys. Rev. E 67, 046224 (2003).

[12] G. Franceschini, S. Bose, and E. Schöll, Phys. Rev. E 60, 5426 (1999).

[13] P. Parmananda, R. Madrigal, M. Rivera, L. Nyikos, I. Z. Kiss, and V. Gáspár, Phys. Rev. E 59, 5266 (1999).

[14] G. Tang, K. He, and G. Hu, Phys. Rev. E 73, 056303 (2006).

[15] K. A. Montgomery and M. Silber, Nonlinearity 17, 2225 (2004).

[16] I. S. Aranson and L. Kramer, Rev. Mod. Phys. 74, 99 (2002).

[17] M. Ipsen, L. Kramer, and P. G. Sørensen, Phys. Rep. 337, 193 (2000).

[18] Y. Kuramoto, Chemical Oscillations, Waves, and Turbulence (Springer, Berlin, 1984).

[19] M. E. Bleich and J. E. S. Socolar, Phys. Rev. E 54, R17 (1996).

[20] I. Harrington and J. E. S. Socolar, Phys. Rev. E 64, 056206
(2001).

[21] C. Postlethwaite and M. Silber, Phys. Rev. D (to be published).

[22] D. Battogtokh, A. Preusser, and A. Mikhailov, Physica D 106, 327 (1997).

[23] D. Battogtokh and A. Mikhailov, Physica D 90, 84 (1996).

[24] S. Boccaletti and J. Bragard, Philos. Trans. R. Soc. London, Ser. A 364, 2383 (2006).

[25] D. V. Ramana Reddy, A. Sen, and G. L. Johnston, Physica D 129, 15 (1999).

[26] O. Beck, A. Amann, E. Schöll, J. E. S. Socolar, and W. Just, Phys. Rev. E 66, 016213 (2002).

[27] J. Unkelbach, A. Amann, W. Just, and E. Schöll, Phys. Rev. E 68, 026204 (2003).

[28] P. C. Matthews, R. E. Mirollo, and S. H. Strogatz, Physica D 52, 293 (1991).

[29] R. E. Mirollo and S. H. Strogatz, J. Stat. Phys. 60, 245 (1990).

[30] F. M. Atay, Phys. Rev. Lett. 91, 094101 (2003).

[31] P. Hövel and E. Schöll, Phys. Rev. E 72, 046203 (2005).

[32] F. Mertens, R. Imbihl, and A. Mikhailov, J. Chem. Phys. 99, 8668 (1993).

[33] M. Falcke and H. Engel, Phys. Rev. E 56, 635 (1997).

[34] M. Stich, M. Ipsen, and A. S. Mikhailov, Physica D 171, 19 (2002).

[35] M. Stich, M. Ipsen, and A. S. Mikhailov, Phys. Rev. Lett. 86, 4406 (2001). 